\title{
Author Correction: Colonial history and global economics distort our understanding of deep-time biodiversity
}

Nussaïbah B. Raja (i), Emma M. Dunne (i), Aviwe Matiwane (D), Tasnuva Ming Khan (D), Paulina S. Nätscher (D), Aline M. Ghilardi (1) and Devapriya Chattopadhyay (1)

Correction to: Nature Ecology \& Evolution https://doi.org/10.1038/s41559-021-01608-8, published online 30 December 2021.

In the version of this article initially published, the designation "Central" America was omitted in an Extended Data Fig. $2 c$ panel heading and the caption as well as in the Extended Data Fig. 3 caption. The figure and captions have been updated to clarify "Central and South America" in each instance.

Published online: 1 February 2022

https://doi.org/10.1038/s41559-022-01682-6

๑ The Author(s), under exclusive licence to Springer Nature Limited 2022 